\title{
Eigenvalue Expansion of Nonsymmetric Linear Compact Operators in Hilbert Space
}

\author{
Ludwig Kohaupt $^{1 *}$
}

\begin{abstract}
For a symmetric linear compact resp. symmetric densely defined linear operator with compact inverse, expansion theorems in series of eigenvectors are known. The aim of the present paper is to generalize the known expansion theorems to the case of corresponding operators without the symmetry property. For this, we replace the set of orthonormal eigenvectors in the symmetric case by a set of biorthonormal eigenvectors resp. principal vectors in the case of simple eigenvalues resp. general eigenvalues. The results for the operators without the symmetry property are all new. Furthermore, if the operators are symmetric, the generalized results deliver the known expansions. As an application of the results for nonsymmetric operators with simple eigenvalues, we obtain a known expansion in a series of eigenfunctions for a non-selfadjoint Boundary Eigenvalue Problem with ordinary differential operator discussed in a book of Coddington/Levinson. But, we obtain a new result if the eigenvalues are general, that is, not necessarily simple. In addition, for a differential operator of 2nd order with constant coefficients, the eigenfunctions and Green's function are explicitly determined. This result is also new, as far as the author is aware.
\end{abstract}

Keywords: Applications to non-selfadjoint boundary eigenvalue problems, Densely defined operator with compact inverse, Eigenvalue expansion of nonsymmetric compact operator, Hilbert space, Nonsymmetric compact operator

2010 AMS: Primary 65J05, Secondary 34L10, 47A10, 47A12

${ }^{1}$ Beuth University of Technology Berlin, Department of Mathematics, Luxemburger Str. 10, 13353 Berlin, Germany, ORCID.

0000-0003-4364-9144

*Corresponding author: kohaupt@beuth-hochschule.de

Received: 28 October 2020, Accepted: 7 April 2021, Available online: 30 June 2021

\section{Introduction}

The paper is structured as follows.

Section 2 is of preparatory nature and of utmost importance for the subsequent sections; it discusses functions of an operator in a Banach space.

Section 3 is on the expansion of a linear compact operator and of a pertinent projection operator in a series of eigenvectors resp. principal vectors in a Hilbert space.

Section 4 treats densely defined linear operators $T=L$ with compact inverse $G=T^{-1}=L^{-1}$, derives for it expansions in series of eigenvectors resp. of principal vectors and shows that $G_{+}=G^{*}$ not only for simple, but also for general eigenvalues, where $G_{+}=L_{+}^{-1}$ and $L_{+}$is the formal adjoint of $L$.

In Section 5, applications of the results of Section 4 are made to a non-selfadjoint BEVP taken from [2, Chapter 12], delivering relation $[2$, Chapter 12, (5.6)]. Here, not only the expansion in a series of eigenfunctions is obtained in the Hilbert 
space $H=L_{2}(a, b)$ if the eigenvalues are simple, but also a corresponding expansion in a series of principal functions if the eigenvalues are general.

In Section 6, beyond this, for a differential operator $L$ defined by $L u(x)=L_{p_{0}, q_{0}} u(x)=-u^{\prime \prime}(x)+p_{0} u^{\prime}(x)+q_{0} u(x), 0 \leq x \leq l$ with real constants $p_{0}$ and $q_{0}$, the eigenvalues $\mu_{j}$ and pertinent eigenfunctions $\chi_{j}(x)$ as well as the associated eigenvalues $\bar{\mu}_{j}=\mu_{j}$ and eigenfunctions $\psi_{j}(x)$ of the formally adjoint operator $L_{+}$defined by $L_{+} v(x)=L_{-p_{0}, q_{0}} v(x)=-v^{\prime \prime}(x)-p_{0} v^{\prime}(x)+q_{0} v(x), 0 \leq$ $x \leq l$ with the biorthonormality property are explicitly determined. Furthermore, the Green's functions $G(x, s)=G\left(x, s ; p_{0}, q_{0}\right)$ pertinent to the operator $L=L_{p_{0}, q_{0}}$ as well as the associated Green's function $G_{+}(x, s)=G^{T}(x, s)=G(s, x)=G\left(s, x ;-p_{0}, q_{0}\right)$ pertinent to the formally adjoint operator $L_{+}=L_{-p_{0}, q_{0}}$ are also explicitly determined confirming the general result $G_{+}=G^{T}$ for the linear compact operators $G$ and $G_{+}$defined by the corresponding Green's functions. In Section 7, we compare the present expansion results in abstract Hilbert spaces with known ones. Finally, Section 8 contains the conclusions.

\section{Functions of an Operator in a Banach Space}

This section contains the basis for the convergence of the studied expansions and is thus of utmost importance for the whole paper.

The method of deriving the expansions for symmetric linear compact operators is no longer applicable when the symmetry property is missing. See, for example the derivation for a symmetric linear compact operator in [14, Theorem 6.4-B, pp.336-337].

A hint what can be done in the nonsymmetric case is found in [2, Chapter 12, 1. Introduction, p.298, first paragraph]. As stated there, an appropriate approach is furnished by the Cauchy integral method. There, one can read: "The method ... yields complete information about the convergence of the expansion for any integrable function."

We mention that most theorems of the classical Theory of Functions can be carried over to functions of a complex variable $z$ with values in a complex Banach space.

So, in particular, Cauchy's integral method can be applied to functions with values in a Banach space, that is, in a complete normed space, where the completeness property of the space is essential.

In [2], the special case of the Hilbert function space $H=L_{2}(a, b)$ is used, that is, a specific complete function space with scalar product.

This is not general enough for our purposes, however. What we need is Cauchy's integral method in a general Banach space. This is treated in the book [6, Chapter I, §5]. However, there Kato assumes that the underlying normed space be finite-dimensional. Then, of course, the space is complete. But, the assumption of finite dimension can be replaced by the completeness of the space since this is the important condition to allow the transition from complex-valued functions of a complex variable to vector-valued functions of a complex variable, as we have already mentioned above. This is done, for instance, in Stummel's paper [13], where Cauchy's integral method is used to show the existence of the resolvent integral for a pair of linear bounded operators $A, B \in B(E, F)$ where $E$ and $F$ are Banach spaces and where it is proven that the completeness property is even not necessary if the operator $B$ is compact.

Here, we study only a single operator $T \in B(E)$, i.e., the pair $(A=T, B=I)$ with the identity operator $I$ in $F=E$ where, for the time being, we assume that the space is complete. In a subsequent paper, we shall investigate whether the completeness property of the space for the series expansion of $T$ can be dropped if $T$ is compact.

For the study of asymptotic expansions for discrete approximations of eigenvalue problems, we refer the reader to [4].

After these preliminary remarks, we turn to functions of an operator in a Banach space as announced in the heading of this section.

We mention that here we use verbatim and almost verbatim passages from [6, Chapter I, $\S 5]$.

Let $\{0\} \neq E$ be a Banach space over the field $\boldsymbol{F}=\mathbb{C}$. Whereas in [6, Chapter I] it is supposed that $\operatorname{dim} E<\infty$, here we assume that $\operatorname{dim} E=\infty$. As already mentioned several times, the following results taken from [6] are valid for $\operatorname{dim} E<\infty$ and $\operatorname{dim} E=\infty$ if the space is complete.

Let $p(\zeta)$ be the polynomial

$$
p(\zeta)=\alpha_{0}+\alpha_{1} \zeta+\cdots \alpha_{n} \zeta^{n}, \zeta \in \mathbb{C}
$$

with $\alpha_{j} \in \mathbb{C}, j=0,1, \cdots, n$. Then the polynomial $p(T) \in B(E)$ is defined by

$$
p(T)=\alpha_{0}+\alpha_{1} T+\cdots \alpha_{n} T^{n}, \zeta \in \mathbb{C},
$$

see [6, Chapter I, §3.3]. Making use of the resolvent

$$
R(\zeta):=(T-\zeta)^{-1}, \zeta \in \mathbb{C},
$$


one can now define the function $\phi(T)$ of $T$ for a more general class of functions $\phi(\zeta)$.

Before we do this, we mention that linear compact operators need not have eigenvalues. For example, Volterra integral operators have no eigenvalues. On the other hand, consider a symmetric linear compact operator. Then, such an operator has at least one eigenvalue, and all eigenvalues are real and simple. It may happen that there exits only a finite number of eigenvalues. Further, there is at most a countable set of eigenvalues with the only possible accumulation point zero, and there exists a set of pertinent pairwise orthonormal eigenvectors. Further, it is known that the non-zero elements of the spectrum consist solely of eigenvalues and that, if there is a countable set of eigenvalues, the assocated sequence tends to zero. For all this, see [14, Chapter 6].

Further, according to [5, Theorem 44.1, p.191], one has $\sigma(T) \backslash\{0\}=\sigma_{P}(T) \backslash\{0\}$ where $\sigma(T)$ is the spectrum of $T$ and $\sigma_{P}(T)$ the point spectrum consisting of the eigenvalues of $T$.

Taking this into account, for our general linear compact operator $T \in B(E)$, we suppose that the spectrum $\sigma(T)$ of $T$ has a countable set of non-zero eigenvalues $\lambda_{j}$ and that the sequence of eigenvalues tends to zero.

Additionally, we suppose that $0 \notin \sigma(T)$ so that $N(T)=\{0\}$ since without this condition, we cannot obtain relation (2.11) resp.(2.14) below.

Now, suppose that $\phi(\zeta)$ is holomorphic in a domain $D$ of the complex plane containing all the eigenvalues $\lambda_{j} \neq 0$ of $T$, and let $C \subset D$ be a simple closed smooth curve with positive direction enclosing all the eigenvalues $\lambda_{j}$ in its interior. Then, $\phi(T)$ is defined by the Dunford-Taylor integral

$$
\phi(T)=-\frac{1}{2 \pi i} \int_{C} \phi(\zeta) R(\zeta) d \zeta=-\frac{1}{2 \pi i} \int_{C} \phi(\zeta)(T-\zeta)^{-1} d \zeta .
$$

This is an analogue of the Cauchy integral formula in the Theory of Functions, see [7, Part I, $\S 15$, p. 61]. More generally, the curve $C$ may consist of several simple closed rectifiable Jordan curves $C_{k}$ having positive direction with interiors $D_{k}^{\prime}$ such that the union of the $D_{k}^{\prime}$ contains all the eigenvalues of $T$. We note that (2.4) does not depend on $C$ as long as $C$ satisfies these conditions. For the $C_{k}$, we can use the circles $C_{k}=\left\{z \in C|| z-\lambda_{k} \mid=r_{k}\right\}$ with sufficiently small radii $r_{k}$.

It can be verified that for the polynomial

$$
\phi(\zeta)=p(\zeta)=\alpha_{0}+\alpha_{1} \zeta+\cdots \alpha_{n} \zeta^{n}, \zeta \in \mathbb{C}
$$

with $\alpha_{j} \in C, j=0,1, \cdots, n$, the Dunford-Taylor integral (2.4) is equal to (2.2).

For the special case

$$
\phi(\zeta)=p(\zeta)=\zeta
$$

we obtain

$$
T=-\frac{1}{2 \pi i} \int_{C} T R(\zeta) d \zeta=T\left(-\frac{1}{2 \pi i} \int_{C} R(\zeta) d \zeta\right)=\left(-\frac{1}{2 \pi i} \int_{C} R(\zeta) d \zeta\right) T .
$$

Now, we set

$$
P:=-\frac{1}{2 \pi i} \int_{C} R(\zeta) d \zeta
$$

According to [6, Chapter I, $\S 5$, Section 3], $P$ is a continuous projection operator onto the algebraic eigenspace $X=P(E)=R(P)$, where $R(P)$ means the range of $P$. Thus, from (2.7) and (2.8), one obtains

$$
T=T P=P T=P T P .
$$

Now, let the radii $r_{k}$ be chosen such that

$$
C_{j} \cap C_{k}=\emptyset, j \neq k, j, k=1,2,3, \cdots .
$$

Then,

$$
P=-\frac{1}{2 \pi i} \int_{C} R(\zeta) d \zeta=\sum_{j=1}^{\infty}\left(-\frac{1}{2 \pi i} \int_{C_{j}} R(\zeta) d \zeta\right)=\sum_{j=1}^{\infty} P_{j}
$$

with

$$
P_{j}=-\frac{1}{2 \pi i} \int_{C_{j}} R(\zeta) d \zeta, j=1,2,3, \cdots
$$


At this point, we needed the assumption $0 \notin \sigma(T)$ since otherwise any circle $C_{0}$ about $\lambda_{0}=0$ would eventually intersect with the circles $C_{k}$ for sufficiently large $k$ so that we would not have (2.10) for $j, k \in(1,2,3, \cdots)$. Let $J$ be the sequence

$$
J:=(1,2,3, \cdots) .
$$

Then, (2.11) can be written as

$$
P=\sum_{j=1}^{\infty} P_{j}=\sum_{j \in J} P_{j} .
$$

Because of (2.10), one has

$$
P_{j} P_{k}=P_{k} P_{j}=P_{j} \delta_{j k}, j, k \in J .
$$

Herewith,

$$
P_{j}(E)=: X_{j}
$$

is the algebraic eigenspace of $T$ associated with the eigenvalue $\lambda_{j}$.

From (2.9), (2.11), and (2.15), we obtain

$$
T=P T=T P=P T P=\sum_{j \in J} P_{j} T=\sum_{j \in J} T P_{j}=\sum_{j \in J} P_{j} T P_{j},
$$

and so

$$
\begin{aligned}
R(T)=T(E) & =(P T)(E)=(T P)(E)=(P T P)(E) \\
& =\sum_{j \in J}\left(P_{j} T\right)(E)=\sum_{j \in J}\left(T P_{j}\right)(E)=\sum_{j \in J}\left(P_{j} T P_{j}\right)(E) .
\end{aligned}
$$

\section{Expansion of a Linear Compact Operator and of a Pertinent Projection Operator in Hilbert Space}

The aim of the present section is to specify the relation (2.17), i.e.,

$$
T=P T=T P=P T P=\sum_{j \in J} P_{j} T=\sum_{j \in J} T P_{j}=\sum_{j \in J} P_{j} T P_{j},
$$

in more detail. This can best be done in a Hilbert space since, for example, the orthogonal projection $P u$ of a vector $u \in H$ onto a unit vector $e \in H$ can be written as

$$
P u=(u, e) e,
$$

that is, by using a scalar product.

In our case, the projection operators $P_{j}$ are not orthogonal, however. But, the dimension of $R\left(P_{j}\right)=P_{j}(H)$ is finitedimensional and represents the geometric eigenspace $N_{j}:=N\left(T-\lambda_{j}\right)$ if the eigenvalue $\lambda_{j}$ is simple and the algebraic eigenspace $X_{j}:=X_{\lambda_{j}}(T)$ if $\lambda_{j}$ is not simple. Now, for finite-dimensional spaces, the author constructed, in earlier work, a set of biorthonormal eigenvectors resp. principal vectors pertinent to a finite-dimensional mapping (usually represented by a matrix with respect to a fixed basis of vectors); here, the mapping is given by $T_{j}=T P_{j}=P_{j} T=P_{j} T P_{j}$. Thus, using these biorthonormal sets, it is possible to specify the expressions $T_{j} u=T P_{j} u=P_{j} T u=P_{j} T P_{j} u$ for elements $u \in H$ in more detail by using a scalar product. This leads to the desired expansion for $T u$. Now, the announced details follow, first for the case of simple eigenvalues, and then for the case of general, not necessarily simple eigenvalues. 


\subsection{The Case of Simple Eigenvalues}

In this subsection, in the case of simple eigenvalues, expansions in a series of eigenvectors are treated; it is organized as follows. First, the conditions for the expansions to hold are stated. Then, the series expansions of $T u$ as well as of $P u$ are derived. Finally, the known expansions for a selfadjoint operator $T=A$ are retrieved from the more general result obtained in this subsection.

(i) The Conditions (C1) - (C4)

We assume the following conditions:

(C1) $\{0\} \neq H$ is a Hilbert space over the field $\boldsymbol{F}=\boldsymbol{C}$ with scalar product

(C2) $0 \neq T \in B(H)$ is compact (or completely continuous) having countably many simple non-zero eigenvalues $\lambda_{1}, \lambda_{2}, \lambda_{3}, \cdots$ with $\lim _{k \rightarrow \infty} \lambda_{k}=0$ pertinent to the eigenvectors $\chi_{1}, \chi_{2}, \chi_{3}, \cdots$. Further, $0 \notin \sigma(T)$.

(C3) The eigenvectors of the adjoint $T^{*}$ of $T$ with the eigenvalues $\bar{\lambda}_{1}, \bar{\lambda}_{2}, \bar{\lambda}_{3}, \cdots$ are $\psi_{1}, \psi_{2}, \psi_{3}, \cdots$

(C4) $\lambda_{i} \neq \lambda_{j}, i \neq j, i, j=1,2,3 \cdots$

(ii) Series Expansions of Tu as well as of Pu

One has the following theorem.

Theorem 3.1 (Biorthonormality relations for $\lambda_{j} \neq \lambda_{k}, j \neq k$ )

Let the conditions $(C 1)-(C 4)$ be fulfilled. Then, with appropriate normalization, the eigenvectors $\chi_{1}, \chi_{2}, \chi_{3}, \cdots$ and $\psi_{1}, \psi_{2}, \psi_{3}, \cdots$ are orthonormal, that is,

$$
\left(\chi_{j}, \psi_{k}\right)=\delta_{j k}, j, k \in J .
$$

Proof: Define the operators

$$
P^{(n)}:=\sum_{j=1}^{n} P_{j}
$$

as well as

$$
T^{(n)}:=T P^{(n)}=\sum_{j=1}^{n} T P_{j}
$$

Here, $R\left(T^{(n)}\right)=\left(T^{(n)}\right)(H)$ is finite-dimensional with dimension $n$. From [8, Theorem1], one has

$$
\left(\chi_{j}, \psi_{k}\right)=\delta_{j k}, j, k=1, \cdots, n .
$$

and

$$
T^{(n)} \chi_{j}=\lambda_{j} \chi_{j}, j=1, \cdots, n .
$$

Now, letting $n \rightarrow \infty$, relation (3.4) entails (3.1) since $T=\lim _{n \rightarrow \infty} T^{(n)}$ according to Section 2 .

Furthermore, we obtain the following theorem.

Theorem 3.2 (Expansions of $T u$ as well as of $P u$ in a series of eigenvectors)

Let the conditions $(\mathrm{Cl})-(\mathrm{C} 4)$ be fulfilled. Then,

$$
T u=\sum_{j \in J} \lambda_{j}\left(u, \psi_{j}\right) \chi_{j}, u \in H
$$

as well as

$$
P u=\sum_{j \in J}\left(u, \psi_{j}\right) \chi_{j}, u \in H .
$$

Proof: Let $u \in H$. Then, due to (3.1),

$$
P^{(n)} u=\sum_{j=1}^{n}\left(u, \psi_{j}\right) \chi_{j}
$$


and thus

$$
T^{(n)} u=T P^{(n)}=\sum_{j=1}^{n} \lambda_{j}\left(u, \psi_{j}\right) \chi_{j} .
$$

Now, from Section 2, the limit

$$
P u=\lim _{n \rightarrow \infty} P^{(n)} u=\sum_{j=1}^{\infty}\left(u, \psi_{j}\right) \chi_{j}=\sum_{j \in J}\left(u, \psi_{j}\right) \chi_{j}
$$

exists entailing also the existence of the limit

$$
T u=\lim _{n \rightarrow \infty} T^{(n)} u=\lim _{n \rightarrow \infty} T P^{(n)} u=\sum_{j=1}^{\infty} \lambda_{j}\left(u, \psi_{j}\right) \chi_{j}=\sum_{j \in J} \lambda_{j}\left(u, \psi_{j}\right) \chi_{j} .
$$

Remark: From (3.6) we conclude that

$$
\overline{\left[\chi_{1}, \chi_{2}, \chi_{3}, \cdots\right]}=T(H)=R(T) .
$$

Further,

$$
P: H \mapsto \overline{\left[\chi_{1}, \chi_{2}, \chi_{3}, \cdots\right]} .
$$

\section{Theorem 3.3}

Let the conditions $(\mathrm{Cl})$ - (C4) be fulfilled. Then, we obtain

$$
u=P u=\sum_{j \in J}\left(u, \psi_{j}\right) \chi_{j}, u \in H
$$

and the projection operator

$$
P_{0}=I-P: H \mapsto N(T)=\{0\} \Longleftrightarrow P_{0}=0 .
$$

Proof: Evidently,

$$
u=P u+(I-P) u, u \in H .
$$

Further,

$$
T\left(P_{0} u\right)=T(I-P) u=T u-T P u=0
$$

where the last equal sign follows from (2.17). So, $P_{0} u \in N(T)=\{0\}$, i.e., $P_{0} u=0, u \in H$ or $P_{0}=0$.

If condition (C4) is not fulfilled, one can remedy this by using a biorthonormalization pre-process, as the next lemma shows.

\section{Lemma 3.4}

Let the conditions (C1) - (C3) be fulfilled, and let, for instance, $\lambda_{j_{1}}, \lambda_{j_{2}}, \cdots, \lambda_{j_{p}}$ be eigenvalues of $T$ with linearly independent eigenvectors $\chi_{j_{1}}, \chi_{j_{2}}, \cdots, \chi_{j_{p}}$; further, let $\psi_{j_{1}}, \psi_{j_{2}}, \cdots, \psi_{j_{p}}$ be linearly independent eigenvectors pertinent to $\bar{\lambda}_{j_{1}}, \bar{\lambda}_{j_{2}}, \cdots, \bar{\lambda}_{j_{p}}$ of $T^{*}$. Then, these eigenvectors can be biorthonormalized such that

$$
\left(\chi_{j_{k}}, \psi_{j_{l}}\right)=\delta_{k l}, k, l=1,2, \cdots, p \text {. }
$$

Proof: See [9, Theorem 3].

After appropriate application of the biorthonormalization pre-process, condition (C4) is satisfied.

(iii) Special Case of a Selfadjoint Compact Operator $T=A$

If $T=A$ is selfadjoint and compact and if there is a countable set of non-zero eigenvalues $\lambda_{j}, j \in J$, then it is known that the relation

$$
\lim _{j \rightarrow \infty} \lambda_{j}=0
$$


is fulfilled. Further, the eigenvalues are real, and the pertinent eigenvectors $\varphi_{j}$ can be chosen real so that one has

$$
\varphi_{j}=\chi_{j}=\psi_{j}, j \in J
$$

meaning that the biorthonormality relations (3.1) turn into the orthonormality relations

$$
\left(\varphi_{j}, \varphi_{k}\right)=\delta_{j, k}, j, k \in J .
$$

Thus, if $0 \notin \sigma(A)$, the relations (3.6) and (3.14) turn into the known results

$$
A u=\sum_{j \in J} \lambda_{j}\left(u, \varphi_{j}\right) \varphi_{j}, u \in H
$$

and

$$
u=P u=\sum_{j \in J}\left(u, \varphi_{j}\right) \varphi_{j}, u \in H
$$

\subsection{The Case of General Eigenvalues}

In this subsection, we do not assume that the eigenvalues of $T$ be simple. Then, we obtain expansions in a series of principal vectors.

This subsection is organized in a similar way as the preceding one.

So, first the conditions for the expansions to hold are stated. Then, the series expansions of $T u$ as well as of $P u$ are derived.

(i) The Conditions $\left(C 1^{\prime}\right)-\left(C 4^{\prime}\right)$

In the general case when the eigenvalues need not be simple, we assume the following conditions:

$\left(C 1^{\prime}\right)\{0\} \neq H$ is a Hilbert space over the field $\boldsymbol{F}=\boldsymbol{C}$ with scalar product $(\cdot, \cdot)$

$\left(C 2^{\prime}\right) 0 \neq T \in B(H)$ is compact (or completely continuous) having countably many non-zero eigenvalues $\lambda_{1}, \lambda_{2}, \lambda_{3}, \cdots$ with $\lim _{k \rightarrow \infty} \lambda_{k}=0$ and the pertinent algebraic eigenspaces $P_{j}(H)=X_{\lambda_{j}}(T)$ spanned by the principal vectors

$\chi_{1}^{(j)}, \chi_{2}^{(j)}, \cdots, \chi_{m_{j}}^{(j)}$ for $j \in J$, where $\chi_{i}^{(j)}$ is of stage $i$. Further, $0 \notin \sigma(T)$.

$\left(C 3^{\prime}\right) \psi_{1}^{(j)}, \psi_{2}^{(j)}, \cdots, \psi_{m_{j}}^{(j)}$ are the principal vectors corresponding to the eigenvalues $\bar{\lambda}_{j}, j \in J$, spanning the algebraic eigenspaces $P_{j}^{*}(H)=X_{\bar{\lambda}_{j}}\left(T^{*}\right)$ for $j \in J$

(C4') $\lambda_{j} \neq \lambda_{k}, j \neq k, j, k \in J$

(ii) Series Expansions of Tu as well as of $\mathrm{Pu}$

As a preparation of the expansions in series of principal vectors, we begin with the detailed biorthonormalization process. According to $\left(C 2^{\prime}\right)$ and $\left(C 3^{\prime}\right)$, we have

$$
T \chi_{k}^{(i)}=\lambda_{i} \chi_{k}^{(i)}+\chi_{k-1}^{(i)}, k=1,2, \cdots, m_{i}
$$

and

$$
T^{*} \psi_{l}^{(j)}=\bar{\lambda}_{j} \psi_{l}^{(j)}+\psi_{l-1}^{(j)}, l=1,2, \cdots, m_{j}
$$

Then, the fact can be used that the principal vectors of stage $k$ are determined only up to a linear combination of principal vectors of stages less than $k$ which was applied in [8] to the chain $\psi_{1}^{(j)}, \psi_{2}^{(j)}, \cdots, \psi_{m_{j}}^{(j)}$ leading to

$$
\left(\chi_{k}^{(i)}, \psi_{l}^{(i)}\right)=0, l \neq m_{i}-k+1, k=1, \cdots, m_{i}
$$

and

$$
\left(\chi_{k}^{(i)}, \psi_{m_{i}-k+1}^{(i)}\right) \neq 0, l=m_{i}-k+1, k=1, \cdots, m_{i} .
$$

So, with

$$
v_{k}^{(i)}=\psi_{m_{i}-k+1}^{(i)},
$$


one has

$$
\left(\chi_{k}^{(i)}, v_{k}^{(i)}\right) \neq 0, k=1, \cdots, m_{i} .
$$

Further, according to [8],

$$
\left(\chi_{k}^{(i)}, v_{l}^{(j)}\right)=0, i \neq j
$$

$k=1, \cdots, m_{i}, l=1, \cdots, m_{j}$.

Now, replace $v_{k}^{(i)}$ in $(3,29)$ by

$$
\tilde{v}_{k}^{(i)}:=\tilde{\psi}_{m_{i}-k+1}:=\beta_{m_{i}-k+1}^{(i)} \psi_{m_{i}-k+1}=\beta_{m_{i}-k+1}^{(i)} v_{k}^{(i)}
$$

and determine the factor $\beta_{m_{i}-k+1}^{(i)}$ such that

$$
\left(\chi_{k}^{(i)}, \tilde{v}_{k}^{(i)}\right)=1 .
$$

Then,

$$
\beta_{m_{i}-k+1}^{(i)}=1 / \overline{\left(\chi_{k}^{(i)}, v_{k}^{(j)}\right)}=1 / \overline{\left(\chi_{k}^{(i)}, \psi_{m_{i}-k+1}\right)}, k=1, \cdots, m_{i}
$$

or

$$
\beta_{l}^{(i)}=1 / \overline{\left(\chi_{m_{i}-l+1}^{(i)}, v_{m_{i}-l+1}^{(j)}\right)}=1 / \overline{\left(\chi_{m_{i}-l+1}^{(i)}, \psi_{l}\right)}, l=1, \cdots, m_{i} .
$$

From (3.31), we obtain

$$
\psi_{l}^{(i)}=\frac{1}{\beta_{l}^{(i)}} \tilde{\psi}_{l}^{(i)}, l=1, \cdots, m_{i}
$$

Inserting this in (3.25) implies

$$
T^{*}\left(\frac{1}{\beta_{l}^{(i)}} \tilde{\Psi}_{l}^{(j)}\right)=\bar{\lambda}_{j}\left(\frac{1}{\beta_{l}^{(i)}} \tilde{\Psi}_{l}^{(j)}\right)+\left(\frac{1}{\beta_{l-1}^{(i)}} \tilde{\Psi}_{l-1}^{(j)}\right), l=1,2, \cdots, m_{j}
$$

or

$$
T^{*} \tilde{\psi}_{l}^{(j)}=\bar{\lambda}_{j} \tilde{\psi}_{l}^{(j)}+\gamma_{l-1}^{(j)} \tilde{\psi}_{l-1}^{(j)}, l=1,2, \cdots, m_{j}
$$

with $\beta_{0}^{(j)}:=1$ and $\tilde{\psi}_{0}^{(j)}:=0$ as well as

$$
\gamma_{l-1}^{(j)}:=\beta_{l}^{(i)} / \beta_{l-1}^{(i)}, l=1,2, \cdots, m_{i} .
$$

This means that in the canonical Jordan form of $T$ restricted to the subspace spanned by the principal vectors $\tilde{\psi}_{1}^{(j)}, \tilde{\psi}_{2}^{(j)}, \cdots, \tilde{\psi}_{m_{j}}^{(j)}$, the ones are to be replaced by the $\gamma_{l-1}^{(j)}, l=2, \cdots, m_{i}$.

Due to the above, one has the following lemma.

Lemma 3.5 (Biorthonormality relations for principal vectors)

Let the conditions $\left(C 1^{\prime}\right)-\left(C 4^{\prime}\right)$ be fulfilled. Then, with the above notations,

$$
\left(\chi_{k}^{(i)}, \tilde{v}_{l}^{(j)}\right)=\delta_{k l} \delta_{i j}
$$

$k=1, \cdots, m_{i}, l=1, \cdots, m_{j}, i, j \in J$ with

$$
\tilde{v}_{l}^{(j)}=\tilde{\psi}_{m_{j}-l+1}^{(j)}=\beta_{m_{j}-l+1}^{(j)} \psi_{m_{j}-l+1}^{(j)}=\beta_{m_{j}-l+1}^{(j)} v_{l}^{(j)},
$$


$l=1, \cdots, m_{j}, j \in J$ as well as

$$
\beta_{m_{j}-l+1}^{(j)}=1 / \overline{\left(\chi_{l}^{(j)}, v_{l}^{(j)}\right)}=1 / \overline{\left(\chi_{l}^{(j)}, \psi_{m_{j}-l+1}\right)}
$$

$l=1, \cdots, m_{j}, j \in J$.

At this point, we mention that

$$
(u, v)=\left(e^{\varphi} u, e^{\varphi} v\right), u, v \in \mathbb{C}^{n}, 0 \leq \varphi<2 \pi
$$

which also applies to the pairs of vectors $u=\chi_{k}^{(i)}, v=\tilde{v}_{l}^{(j)}$ in (3.38).

Remark: We note that the matrix

$$
\left(\left(\chi_{k}^{(i)}, \tilde{\psi}_{l}^{(i)}\right)\right)_{k, l=1, \cdots, m_{i}}
$$

has the form

$$
\left[\begin{array}{llll} 
& & & 1 \\
& & 1 & \\
& \vdots & & \\
1 & & &
\end{array}\right]
$$

which is called cross-diagonal in [12, p.3] and anti-diagonal by other authors. As opposed to this, the matrix $\left(\left(\chi_{k}^{(i)}, \tilde{v}_{l}^{(i)}\right)\right)_{k, l=1, \cdots, m_{i}}$, is equal to the identity matrix and thus diagonal.

With Lemma 3.5, we can derive the next theorem that is an analogue to Theorem 3.2.

Theorem 3.6

Let the conditions $\left(C 1^{\prime}\right)-\left(C 4^{\prime}\right)$ be fulfilled. Then,

$$
T u=\sum_{j \in J} \sum_{k=1}^{m_{j}}\left(u, \tilde{v}_{k}^{(j)}\right)\left[\lambda_{j} \chi_{k}^{(j)}+\chi_{k-1}^{(j)}\right], u \in H
$$

as well as

$$
P u=\sum_{j \in J} \sum_{k=1}^{m_{j}}\left(u, \tilde{v}_{k}^{(j)}\right) \chi_{k}^{(j)}, u \in H .
$$

Proof: Define

$$
P^{(n)} u=\sum_{j=1}^{n} P_{j}=\sum_{j=1}^{n} P_{\lambda_{j}}(T) .
$$

Since $P^{(n)}(H)$ is finite-dimensional, Lemma 3.4 entails

$$
P^{(n)} u=\sum_{j=1}^{n} \sum_{k=1}^{m_{j}}\left(u, \tilde{v}_{k}^{(j)}\right) \chi_{k}^{(j)}, u \in H
$$

This leads to

$$
\begin{aligned}
T^{(n)} u: & =T P^{(n)} u=\sum_{j=1}^{n} \sum_{k=1}^{m_{j}}\left(u, \tilde{v}_{k}^{(j)}\right) T \chi_{k}^{(j)} \\
& =\sum_{j=1}^{n} \sum_{k=1}^{m_{j}}\left(u, \tilde{v}_{k}^{(j)}\right)\left[\lambda_{j} \chi_{k}^{(j)}+\chi_{k-1}^{(j)}\right], u \in H .
\end{aligned}
$$

From this, it follows, based on Section 2,

$$
P=\lim _{n \rightarrow \infty} P^{(n)}
$$


as well as

$$
T=\lim _{n \rightarrow \infty} T^{(n)}
$$

in the Banach space $B(H)$. From (3.45) - (3.49), the relations (3.43) and (3.44) follow.

Using (3.44), we obtain the next theorem.

Theorem 3.7

Let the conditions $\left(C 1^{\prime}\right)-\left(C 4^{\prime}\right)$ be fulfilled. Then,

$$
u=P u=\sum_{j \in J} \sum_{k=1}^{m_{j}}\left(u, \tilde{v}_{k}^{(j)}\right) \chi_{k}^{(j)}, u \in H .
$$

Proof: The proof is done in the same way as for Theorem 3.3

Remark: As in the case of simple eigenvalues of $T$, under the conditions $\left(C 1^{\prime}\right)-\left(C 4^{\prime}\right)$ the relation $N(T)=\{0\}$ is equivalent to the property that $\lambda_{0}=0$ is not an eigenvalue of $T$ which, in turn, is equivalent to $\bar{\lambda}_{0}=0$ is not an eigenvalue of $T^{*}$ or that $N\left(T^{*}\right)=\{0\}$.

Remark: If condition $\left(C 4^{\prime}\right)$ is not fulfilled, this again can be remedied by a biorthonormalization pre-process described in [9, Theorem 4].

\section{Series Expansions for a Densely Defined Linear Operator with Compact Inverse}

The results on linear compact operators in Section 3 can be carried over to densely defined linear operators with compact inverse. The obtained expansions have important applications to BEVPs for ordinary and partial differential equations, where in Section 5, we restrict ourselves to BEVPs for ODEs. Again, it is natural to first handle the case of simple eigenvalues and then the case of general eigenvalues.

\subsection{The Case of Simple Eigenvalues}

In this subsection, in the case of simple eigenvalues, expansions in series of eigenvectors are treated.

It is structured as follows. We begin with the conditions on the densely defined linear operator $L$, its formally adjoint operator $L_{+}$and their pertinent compact inverses $G$ and $G_{+}$. Then, it is shown that $G_{+}=G^{*}$ where $G^{*}$ is the adjoint operator of $G$. Next, the expansions for $G u$ and $P u$ in series of eigenvectors are derived.

(i) The Conditions $\left(C 1_{d}\right)-\left(C 5_{d}\right)$

We assume the following conditions:

$\left(C 1_{d}\right)\{0\} \neq H$ is a Hilbert space over the field $\boldsymbol{F}=\boldsymbol{C}$ with scalar product $(\cdot, \cdot)$

$\left(C 2_{d}\right)\{0\} \neq H_{D}$ and $H_{R}$ are pre-Hilbert spaces with

$$
H_{D} \subset H_{R} \subset H, \bar{H}_{D}=\bar{H}_{R}=H
$$

and where

$$
L: D(L):=H_{D} \mapsto H_{R}
$$

is a linear operator with the countably many simple non-zero eigenvalues $\mu_{1}, \mu_{2}, \mu_{3}, \cdots$ and the property $\lim _{j \rightarrow \infty} \mu_{j}=\infty$ as well as pertinent eigenvectors $\chi_{1}, \chi_{2}, \chi_{3}, \cdots \in H_{D}$. Further, $L$ possesses a compact inverse

$$
G:=L^{-1} \in B(H)
$$

$\left(C 3_{d}\right)\{0\} \neq H_{D,+}$ and $H_{R}$ are pre-Hilbert spaces with

$$
H_{D,+} \subset H_{R} \subset H, \bar{H}_{D,+}=\bar{H}_{R}=H
$$

and where

$$
L_{+}: D\left(L_{+}\right):=H_{D,+} \mapsto H_{R}
$$

is a linear operator with the countably many simple non-zero eigenvalues $\mu_{1,+}, \mu_{2,+}, \mu_{3,+}, \cdots$ and the property $\lim _{j \rightarrow \infty} \mu_{j,+}=\infty$ as well as pertinent eigenvectors $\psi_{1}, \psi_{2}, \psi_{3}, \cdots \in H_{D,+}$. Further, $L_{+}$possesses a compact inverse

$$
G_{+}:=L_{+}^{-1} \in B(H)
$$


$\left(C 4_{d}\right)(L u, v)=\left(u, L_{+} v\right), u \in H_{D}, v \in H_{D,+}$

$\left(C 5_{d}\right) \mu_{j} \neq \mu_{k}, j \neq k, j, k \in J$

We mention that due to the above conditions, $0 \notin \sigma(G)$.

(ii) Series Expansions of $G u$ and $P u$

The first theorem reads as follows.

Theorem 4.1

Let the conditions $\left(C 1_{d}\right)-\left(C 5_{d}\right)$ be fulfilled. Then,

$$
\mu_{j,+}=\bar{\mu}_{j}, j \in J
$$

and

$$
G_{+}=G^{*}
$$

where $G^{*} \in B(H)$ is the adjoint operator of $G$ defined by

$$
(G u, v)=\left(u, G^{*} u\right), u, v \in H .
$$

Further, the operator $G$ has the eigenvalues $\lambda_{j}=1 / \mu_{j}$ as well as the eigenvectors $\chi_{j}$, and $G_{+}=G^{*}$ has the eigenvalues $\lambda_{j,+}=\bar{\lambda}_{j}=1 / \mu_{j,+}=1 / \bar{\mu}_{j}$ as well as the eigenvectors $\psi_{j}$ for $j \in J$. In addition, $\lim _{j \rightarrow \infty} \lambda_{j}=0$.

Proof: Let $\tilde{u}, \tilde{v} \in H_{R}$ and

$$
u:=L^{-1} \tilde{u}=G \tilde{u}
$$

as well as

$$
v:=L_{+}^{-1} \tilde{v}=G_{+} \tilde{v} .
$$

Then,

$$
u \in H_{D}, v \in H_{D,+} .
$$

Substituting this in $\left(C 4_{d}\right)$ gives

$$
\left(\tilde{u}, G_{+} \tilde{v}\right)=(G \tilde{u}, \tilde{v}), \tilde{u}, \tilde{v} \in H_{R}
$$

or, with new denotations,

$$
\left(u, G_{+} v\right)=(G u, v), u, v \in H_{R},
$$

i.e.,

$$
(G u, v)=\left(u, G_{+} v\right), u, v \in H_{R},
$$

and thus, because of $\bar{H}_{R}=H$, also

$$
(G u, v)=\left(u, G_{+} v\right), u, v \in H .
$$

On the other hand,

$$
(G u, v)=\left(u, G^{*} v\right), u, v \in H
$$

and consequently

$$
G_{+}=G^{*} .
$$

The rest of the proof is obtained in a simple way.

From Theorem 4.1 and the results of Subsection 3.1, we obtain the following corollary. 


\section{Corollary 4.2}

Let the conditions $\left(C 1_{d}\right)-\left(C 5_{d}\right)$ be fulfilled. Then,

$$
\begin{aligned}
& G u=\sum_{j \in J} \lambda_{j}\left(u, \psi_{j}\right) \chi_{j}, u \in H, \\
& u=P u=\sum_{j \in J}\left(u, \psi_{j}\right) \chi_{j}, u \in H .
\end{aligned}
$$

Proof: Because of

$$
G \chi_{j}=\lambda_{j} \chi_{j}
$$

and

$$
G^{*} \psi_{j}=G_{+} \psi_{j}=\lambda_{j,+} \psi_{j}=\bar{\lambda}_{j} \psi_{j},
$$

$j \in J$, from Section 3.1 we obtain the relations (4.4) and (4.5).

\subsection{The Case of General Eigenvalues}

In this subsection, we do not assume that the eigenvalues of $L$ be simple. Then, we obtain expansions in a series of principal vectors.

This subsection is organized in a similar way as the preceding one.

So, first the conditions on the densely defined linear operator $L$, its formally adjoint operator $L_{+}$and their compact inverses $G$ and $G_{+}$are stated. Next, the expansions of $G u$ and $P u$ in series of principal vectors are derived.

(i) The Conditions $\left(C 1_{d}^{\prime}\right)-\left(C 5_{d}^{\prime}\right)$

We assume the following conditions:

$\left(C 1_{d}^{\prime}\right)\{0\} \neq H$ is a Hilbert space over the field $\boldsymbol{F}=\boldsymbol{C}$ with scalar product $(\cdot, \cdot)$

$\left(C 2_{d}^{\prime}\right)\{0\} \neq H_{D}$ and $H_{R}$ are pre-Hilbert spaces with

$$
H_{D} \subset H_{R} \subset H, \bar{H}_{D}=\bar{H}_{R}=H
$$

and where

$$
L: D(L):=H_{D} \mapsto H_{R}
$$

is a linear operator with the countably many general non-zero eigenvalues $\mu_{1}, \mu_{2}, \mu_{3}, \cdots$ and the property $\lim _{j \rightarrow \infty} \mu_{j}=\infty$ as well as pertinent principal vectors $\chi_{1}^{(j)}, \chi_{2}^{(j)}, \cdots \chi_{m_{j}}^{(j)} \in H_{D} j \in J$, where $\chi_{i}^{(j)}$ is of stage $i$. Further, $L$ possesses a compact inverse

$$
G:=L^{-1} \in B(H)
$$

$\left(C 3_{d}^{\prime}\right)\{0\} \neq H_{D,+}$ and $H_{R}$ are pre-Hilbert spaces with

$$
H_{D,+} \subset H_{R} \subset H, \bar{H}_{D,+}=\bar{H}_{R}=H
$$

and where

$$
L_{+}: D\left(L_{+}\right):=H_{D,+} \mapsto H_{R}
$$

is a linear operator with the countably many general non-zero eigenvalues

$\mu_{1,+}, \mu_{2,+}, \mu_{3,+}, \cdots$ and the property $\lim _{j \rightarrow \infty} \mu_{j,+}=\infty$ as well as pertinent principal vectors $\psi_{1}^{(j)}, \psi_{2}^{(j)}, \cdots \psi_{m_{j}}^{(j)} \in H_{D,+} j \in$ $J$. Further, $L_{+}$possesses a compact inverse

$$
G_{+}:=L_{+}^{-1} \in B(H)
$$


$\left(C 4_{d}^{\prime}\right)(L u, v)=\left(u, L_{+} v\right), u \in H_{D}, v \in H_{D,+}$

$\left(C 5_{d}^{\prime}\right) \mu_{j} \neq \mu_{k}, j \neq k, j, k \in J$

Again, we mention that due to the above conditions, $0 \notin \sigma(G)$.

(ii) Series Expansions of $G u$ and $P u$

The next theorem reads as follows.

Theorem 4.3

Let the conditions $\left(C 1_{d}^{\prime}\right)-\left(C 5_{d}^{\prime}\right)$ instead of $\left(C 1_{d}\right)-\left(C 5_{d}\right)$ be fulfilled. Then, the relations (4.1) - (4.3) as well as $\lim _{j \rightarrow \infty} \lambda_{j}=0$ of Theorem 4.1 hold.

Proof: The proof of Theorem 4.3 is the same as for Theorem 4.1 since it does not depend on the condition that the eigenvalues be simple.

From Theorem 4.3 and the results of Subsection 3.2, we obtain the following corollary.

Corollary 4.4

Let the conditions $\left(C 1_{d}^{\prime}\right)-\left(C 5_{d}^{\prime}\right)$ be fulfilled. Then,

$$
\begin{aligned}
& G u=\sum_{j \in J} \sum_{k=1}^{m_{j}}\left(u, \tilde{v}_{k}^{(j)}\right)\left[\lambda_{j} \chi_{k}^{(j)}+\chi_{k-1}^{(j)}\right], u \in H, \\
& u=P u=\sum_{j \in J} \sum_{k=1}^{m_{j}}\left(u, \tilde{v}_{k}^{(j)}\right) \chi_{k}^{(j)}, u \in H .
\end{aligned}
$$

\section{Application to a General Non-Selfadjoint BEVP with Ordinary Differential Operator of nth Order}

In this section, we apply the results of Section 4 to a general non-selfadjoint BEVP for an ordinary differential operator $L$ of nth order. In doing so, we not only obtain the expansion (1.7) for simple eigenvalues, but also, in addition, those for $P u$ and $G u$ in series of eigenfunctions, and further those for general eigenvalues in series of principal functions, which is much more than what is obtained in [2] before.

We mention that this section contains a series of verbatim and almost verbatim passages from [2, Chapter 11].

Now, the details follow.

Let $a \leq x \leq b$ be a closed bounded interval, and let $L$ be the linear differential operator of nth order with $n \geq 1$ defined by

$$
(L u)(x):=a_{n}(x) u^{(n)}(x)+a_{n-1}(x) u^{(n-1)}(x)+\cdots+a_{1}(x) u^{\prime}(x)+a_{0}(x) u(x)
$$

where $a_{k}$ are complex-valued functions of class $C^{k}[a, b]$ and $a_{n}(x) \neq 0$ on $[a, b]$. Given any set of $2 m n$ complex constants $\alpha_{i j}, \beta_{i j}, i=1,2, \cdots, m, j=0,1, \cdots, n-1$, define the $m$ boundary operators or boundary forms $R_{1}, \cdots, R_{m}$ for the functions $u$ on $[a, b]$, for which $u^{(j)}, j=1,2, \cdots, n$ exist at $a$ and $b$ by

$$
R_{i} u:=\sum_{j=0}^{n-1}\left\{\alpha_{i j} u^{(j)}(a)+\beta_{i j} u^{(j)}(b)\right\}=0, i=1,2, \cdots, m
$$

$$
R u=0 .
$$

We suppose that $R$ has rank $m$. Corresponding to any homogeneous boundary value problem (for short: BVP) is a well-defined "adjoint" problem (which should better be called formally adjoint problem) with the Lagrange "adjoint operator" given by

$$
\begin{aligned}
\left(L_{+} v\right)(x)=(-1)^{n}\left(\bar{a}_{n}(x) v\right)^{(n)}(x)+ & (-1)^{n-1}\left(\bar{a}_{n-1}(x) v\right)^{(n-1)}(x)+\cdots \\
& +(-1)\left(\bar{a}_{1}(x) v\right)^{\prime}(x)+\bar{a}_{0}(x) v(x)
\end{aligned}
$$

and a set of adjoint boundary conditions

$$
R_{+} v=0
$$


complementary in a sense to those for the problem pertinent $L$.

We mention that some authors denote the formally adjoint operator by $L^{*}$, see for instance [10]. But, we do not follow this usage since this paper is functional-analysis-oriented and since $L^{*}$ could be misinterpreted as the adjoint of a densely defined linear operator $L$, see [1, No.44]. Instead, as in [2], we use a plus sign to denote the formally adjoint operator, here as a subscript instead of a superscript there.

We note that an adjoint boundary condition is not unique, see [2, Theorem 2.1].

Now, we define the pre-Hilbert spaces

$$
H_{D}:=D(L):=\left\{u \in C^{n}[a, b] \mid R u=0\right\}
$$

and

$$
H_{D,+}:=D\left(L_{+}\right):=\left\{v \in C^{n}[a, b] \mid R_{+} v=0\right\} .
$$

Then,

$$
(L u, v)=\left(u, L_{+} v\right), u \in H_{D}, v \in H_{D,+} .
$$

We mention that

$$
C_{0}^{\infty}[a, b] \subset H_{D} \subset H_{R}:=C_{2}[a, b] \subset L_{2}(a, b)=: H
$$

where $C_{2}[a, b]$ is the function space $C[a, b]$ endowed with the norm

$$
\|u\|_{2}=\left(\int_{a}^{b}|u(x)|^{2} d x\right)^{\frac{1}{2}},
$$

and where the integral is taken in the sense of Riemann which is equal to the Lebesgue integral for $u \in C_{2}[a, b]$. The space $L_{2}(a, b)$ is the space of measurable functions such that the above integral (taken in the sense of Lebesgue) is finite.

Corresponding to (5.9), one has

$$
C_{0}^{\infty}[a, b] \subset H_{D,+} \subset H_{R}=C_{2}[a, b] \subset L_{2}(a, b)=H .
$$

It is known that

$$
\overline{C_{0}^{\infty}[a, b]}=L_{2}(a, b) .
$$

If $R$ is a boundary form of rank $m$, the problem

$$
\pi_{m}: L u=0, u \in H_{D}=D(L)
$$

is called a homogeneous BVP of rank $m$.

The problem

$$
\pi_{2 n-m,+}: L_{+} v=0, v \in H_{D,+}=D\left(L_{+}\right)
$$

is called the adjoint $B V P$.

One has the following:

$\pi_{n}$ and $\pi_{n,+}$ have the same number of independent solutions. see [2, p.293, last line].

The BEVP pertinent to $\pi_{n}$ is given by

$$
\pi_{n, \mu}: L u=\mu u, u \in H_{D}=D(L)
$$

and that associated with $\pi_{n,+}$ by

$$
\pi_{n, \bar{\mu},+}: L_{+} v=\bar{\mu} v, v \in H_{D,+}=D\left(L_{+}\right) .
$$

Now, let $G(x, s)$ be the Green's function pertinent to the BVP $\pi_{n}$ and $G_{+}(x, s)$ the Green's function associated with $\pi_{n,+}$. Then, the pertinent compact operators $G=L^{-1}$ and $G_{+}=L_{+}^{-1}$ are given by

$$
(G u)(x)=\int_{a}^{b} G(x, s) u(s) d s, u \in L_{2}(a, b)
$$


and

$$
\left(G_{+} v\right)(x)=\int_{a}^{b} G_{+}(x, s) v(s) d s, v \in L_{2}(a, b)
$$

where

$$
G_{+}(x, s)=\overline{G(s, x)}, x, s \in[a, b],
$$

see [2, (4.15)] implying for the pertinent operators $G$ in (5.15) and $G_{+}$in (5.17) the relations

$$
G_{+}=G^{*} .
$$

If the conditions $\left(C 1_{d}\right)-\left(C 5_{d}\right)$ for $L$ in (5.1) and for $L_{+}$in (5.4) are fulfilled, then (5.19) follows also from the abstract results of Section 4, and beyond this, one obtains also the expansions in series of eigenvectors (4.4) and (4.5) in Corollary 4.2 with convergence in the norm $\|\cdot\|_{2}$, whereas in $[2$, Chapter 12,(5.6)] only the relation (4.5), i.e.,

$$
u=\sum_{j=1}^{\infty}\left(u, \psi_{j}\right) \chi_{j}, u \in H=L_{2}(a, b)
$$

is given.

Beyond this, if the conditions $\left(C 1_{d}^{\prime}\right)-\left(C 5_{d}^{\prime}\right)$ are fulfilled, then the expansions in series of principal vectors (4.6) and (4.7) are valid in the norm $\|\cdot\|_{2}$. This case when the eigenvalues are general is not treated in [2] and means a considerable progress in the theory of non-selfadjoint BEVPs.

\section{The Case of a Non-Selfadjoint BEVP of 2 nd Order}

In this section, we further specialize the BEVP discussed in Section 5 by restricting the order of $L$ to $n=2$ and by employing very simple boundary values. The considered problem is often used as an example in books on Mathematical Physics and is treated there in a special weighted norm. But when it comes to specific examples, the term with the first derivative usually is omitted so that one obtains a selfadjoint problem. Here, we keep this term, and so we get a non-selfadjoint problem of 2 nd order.

This section is split up in two subsections.

In Subsection 6.1, the BEVP of 2nd order with real continuous coefficients is established. It goes without saying that the series expansions obtained in Section 5 are valid if the corresponding conditions are fulfilled.

In Subsection 6.2, we further specialize the BEVP of 2nd order to the case when the coefficients are constant. Then, it is possible to explicitly determine the eigenvalues, biorthonormal eigenfunctions, and the Green's functions defining the inverse operators $G$ of $L$ and $G_{+}$of $L_{+}$.

\subsection{The BEVP of 2nd Order with Real Continuous Coefficients}

As a special case of the general differential operator of nth order in Section 5, in this subsection we consider the differential operator of 2 nd order

$$
L u(x):=a_{2}(x) u^{\prime \prime}(x)+a_{1}(x) u^{\prime}(x)+a_{0}(x) u(x), 0 \leq x \leq l
$$

with real functions $a_{i} \in C^{i}[0, l], i=0,1,2$ and the boundary conditions

$$
R u=0 \Longleftrightarrow u(0)=u(l)=0,
$$

cf. e.g., $\left[11, \S 75\right.$, p.362] where $a_{2}(x)=-1, a_{1}(x)=p(x), a_{0}(x)=q(x), l=1$.

We mention that we have chosen here the interval $[0, l]$ since, in applications to mechanical problems, $l$ means a length.

The formally adjoint operator $L_{+}$reads

$$
L_{+} v(x):=\left(a_{2}(x) v\right)^{\prime \prime}(x)-\left(a_{1}(x) v\right)^{\prime}(x)+a_{0}(x) v(x), 0 \leq x \leq l .
$$

As adjoint boundary condition, we choose

$$
R_{+} v=0 \Longleftrightarrow v(0)=v(l)=0
$$


so that $R_{+} v=R u=0$ holds. Here, we have

$$
H_{D}=\left\{u \in C^{2}[0, l] \mid u(0)=u(l)=0\right\}=H_{D,+}
$$

and

$$
H_{R}=C_{2}[0, l]
$$

as well as

$$
H=L_{2}(0, l) \text {. }
$$

Herewith,

$$
(L u, v)=\left(u, L_{+} v\right), u \in H_{D}, v \in H_{D,+}
$$

so that condition $\left(C 4_{d}\right)$ is fulfilled.

We further suppose that the differential operator $L$ in (6.1) has a countable set of simple non-zero eigenvalues $\mu_{1}, \mu_{2}, \mu_{3}, \cdots$ with $\lim _{j \rightarrow \infty} \mu_{j}=\infty$. Then, the conditions $\left(C 1_{d}\right)-\left(C 5_{d}\right)$ are fulfilled, and one has the expansions in series of eigenfunctions (4.4) and (4.5).

\subsection{The Special Case of Constant Coefficients}

In this subsection, we treat the BEVP of Subsection 6.1 when $a_{2}(x)=-1, a_{1}(x)=p(x)=p_{0}, a_{0}(x)=q(x)=q_{0}$ are constant in the interval $[0, l]$, that is, when $L u=-u^{\prime \prime}+p_{0} u^{\prime}+q_{0} u$ and thus $L_{+} v=-v^{\prime \prime}-p_{0} v^{\prime}+q_{0} v$.

In this special case, it is possible to explicitly determine the eigenvalues $\mu_{j}$ of $L$ resp. $\mu_{j}$ of $L_{+}$and the pertinent eigenfunctions $\chi_{j}$ resp. $\psi_{j}$, as the case may be. Further, the Green's functions $G\left(x, s ; p_{0}, q_{0}\right)$ and $G_{+}\left(x, s ; p_{0}, q_{0}\right)$ defining the inverse compact operators $G$ and $G_{+}=G^{T}$ are explicitly determined. As far as the author is aware, these results have not been obtained, before.

For the sake of brevity, the details of the derivation of these quantities are left to the reader. However, we give some hints for obtaining these results.

(i) The Differential Operators $L$ and $L_{+}$and Pertinent BEVPs

As already announced, in this subsection, we choose constant coefficients in the differential operator $L$. More precisely, we set

$$
a_{2}(x)=-1, a_{1}(x)=p(x)=p_{0}, a_{0}(x)=q(x)=q_{0}
$$

with real constants $p_{0}$ and $q_{0}$ so that

$$
(L u)(x)=-u^{\prime \prime}(x)+p_{0} u^{\prime}(x)+q_{0} u(x), 0 \leq x \leq l
$$

and

$$
\left(L_{+} v\right)(x)=-v^{\prime \prime}(x)-p_{0} v^{\prime}(x)+q_{0} v(x), 0 \leq x \leq l
$$

with the same boundary conditions (6.2) and (6.4) as in Subsection 6.1.

We restrict the constant $q_{0}$ to $q_{0}>0$.

The pertinent BEVPs read

$$
\pi_{2, \mu}: L u=\mu u, u \in H_{D}=D(L)
$$

and that associated with $\pi_{2,+}$ by

$$
\pi_{2, \bar{\mu},+}: L_{+} v=\bar{\mu} v, v \in H_{D,+}=D\left(L_{+}\right) .
$$

(ii) The Eigenvalues and Eigenfunctions

The eigenvalues of $L$ and $L_{+}$are given by

$$
\mu=\bar{\mu}=\mu_{j}=\bar{\mu}_{j}=\frac{j^{2} \pi^{2}}{l^{2}}+D, j \in J
$$


with the quantity

$$
D=D\left(p_{0}, q_{0}\right)=\left(\frac{p_{0}}{2}\right)^{2}+q_{0}>0
$$

so that

$$
\lim _{j \rightarrow \infty} \lambda_{j}=\lim _{j \rightarrow \infty} \frac{1}{\mu_{j}}=0
$$

is fulfilled.

The biorthonormal eigenfunctions are found to be

$$
\chi_{j}(x)=\sqrt{\frac{2}{l}} \exp \left(\frac{p_{0}}{2} x\right) \sin j \pi \frac{x}{l}, 0 \leq x \leq l, j \in J
$$

and

$$
\psi_{k}(x)=\sqrt{\frac{2}{l}} \exp \left(-\frac{p_{0}}{2} x\right) \sin k \pi \frac{x}{l}, 0 \leq x \leq l, k \in J
$$

so that we have

$$
\left(\chi_{j}, \psi_{k}\right)=\int_{0}^{l} \chi_{j}(x) \psi_{k}(x) d x=\frac{2}{l} \int_{0}^{l} \sin \left(j \pi \frac{x}{l}\right) \sin \left(k \pi \frac{x}{l}\right) d x=\delta_{j k}, j, k \in J .
$$

Hint: To derive these results, use the ansatz $u(x)=c e^{\kappa x}$ in order to solve the BEVP

$$
L_{p_{0}, q_{0}} u=\mu u, u(0)=u(l)=0 .
$$

The eigenfunctions $\psi_{j}(x)$ are obtained from $\chi_{j}(x)$ by just replacing $p_{0}$ by $-p_{0}$.

(iii) The Green's Function of $L_{p_{0}, q_{0}} u=0, u(0)=u(l)=0$

A set of fundamental solutions of the BVP $L_{p_{0}, q_{0}} u=0, u(0)=u(l)=0$, i.e., when $\mu=0$, is given by

$$
\begin{aligned}
& u_{1}(x)=\exp \left(\frac{p_{0}}{2} x\right) \sinh \sqrt{D} x, 0 \leq x \leq l, \\
& u_{2}(x)=\exp \left(\frac{p_{0}}{2} x\right) \cosh \sqrt{D} x, 0 \leq x \leq l,
\end{aligned}
$$

with

$$
D=D\left(p_{0}, q_{0}\right)=\left(\frac{p_{0}}{2}\right)^{2}+q_{0}
$$

which is also obtained with the ansatz $u(x)=c e^{\kappa x}$ by setting $c=1$ and taking into account $\mu=0$ where here $D$ is a discriminant.

Based on these fundamental solutions, we have calculated the Green's functions by the method described in [10, pp.311].

Thus, one gets

$$
G(x, s)=\left\{\begin{array}{l}
G_{1}(x, s)=\frac{\sinh \sqrt{D} x \sinh \sqrt{D}(l-s)}{\sqrt{D} \sinh \sqrt{D} l} \exp \left(\frac{p_{0}}{2}(x-s)\right), 0 \leq x \leq s \leq l, \\
G_{2}(x, s)=\frac{\sinh \sqrt{D}(l-x) \sinh \sqrt{D} s}{\sqrt{D} \sinh \sqrt{D} l} \exp \left(\frac{p_{0}}{2}(x-s)\right) 0 \leq s \leq x \leq l .
\end{array}\right.
$$

For $G_{+}(x, s)$, we obtain

$$
G_{+}(x, s)=\left\{\begin{array}{l}
G_{+, 1}(x, s)=\frac{\sinh \sqrt{D}(l-x) \sinh \sqrt{D} s}{\sqrt{D} \sinh \sqrt{D} l} \exp \left(\frac{p_{0}}{2}(s-x)\right), 0 \leq x \leq s \leq l, \\
G_{+, 2}(x, s)=\frac{\sinh \sqrt{D} x \sinh \sqrt{D}(l-s)}{\sqrt{D} \sinh \sqrt{D} l} \exp \left(\frac{p_{0}}{2}(s-x)\right) 0 \leq s \leq x \leq l .
\end{array}\right.
$$


so that, because of $D=D\left(p_{0}, q_{0}\right)$,

$$
G(x, s)=G\left(x, s ; p_{0}, q_{0}\right)
$$

and

$$
G_{+}(x, s)=G^{T}(x, s)=G(s, x)=G\left(s, x ;-p_{0}, q_{0}\right)
$$

in accordance with the fact that, for the pertinent operators, one has $G_{+}=G^{T}$.

\section{Comparison of Present Expansion Results with Known Ones in an Abstract Hilbert Space}

The oldest expansion result for compact operators in an abstract Hilbert space being of formal similarity to our results the author found is that in [1, Section 64, pp.172-174]. There, under certain conditions, the expansions of the form

$$
h=h_{0}+\sum_{j \in J}\left(h, e_{j}\right) e_{j}, \quad h \in H
$$

with an element $h_{0} \in H_{0}:=N(T)$ as well as

$$
T h=\sum_{j \in J} \mu_{j}\left(h, e_{j}\right) g_{j}, \quad h \in H
$$

can be found. Here, the vectors $e_{j}$ are the pairwise orthonormal eigenvectors of $A:=T^{*} T$. The associated eigenvalues $\lambda_{j}$ can be written in the form

$$
\lambda_{j}=\left(A e_{j}, e_{j}\right)=\left(T^{*} T e_{j}, e_{j}\right)=\left(T e_{j}, T e_{j}\right)>0 .
$$

Therefore, one has $\lambda_{j}=\mu_{j}^{2}$, where $\mu_{1} \geq \mu_{2} \geq \cdots>0$.

The vectors $g_{j}$ are defined by

$$
T e_{j}=\mu_{j} g_{j}
$$

leading to

$$
\left(g_{j}, g_{k}\right)=\delta_{j k} .
$$

Applying $T$ to (7.1) and using (7.4), we obtain (7.2).

As opposed to this, our result is an expansion in series of eigenvalues and eigenvectors/principal vectors of the compact operator $T$ itself whereas in [1] one has an expansion in series of eigenvalues $\mu_{j}=\mu_{j}\left(T^{*} T\right)$ and eigenvectors $e_{j}=e_{j}\left(T^{*} T\right)$ of $T^{*} T$ and the vectors $g_{j}$ defined in (7.4) that are left singular vectors in the denotation of [3, p.2].

The most recent publication on expansions of a compact operator in an abstract Hilbert space the author has found is [3]. There, it is used that the singular values and singular vectors of $T$ are related to the nonzero eigenvalues and corresponding eigenvectors of $T^{*} T$ and $T T^{*}$. More precisely, one has

$$
T \phi_{k}=\sigma_{k} \psi_{k},
$$

$$
T^{*} T \phi_{k}=\sigma_{k}^{2} \phi_{k},
$$

$$
T T^{*} \psi_{k}=\sigma_{k}^{2} \psi_{k} .
$$

The quantities $\sigma_{k}$ are called singular values, the vectors $\phi_{k}$ are called right singular vectors and $\psi_{k}$ left singular vectors in [3, p.2]. Herewith, it is proven that the expansion

$$
T=\sum_{k=1}^{\infty} \sigma_{k} \psi_{k} \otimes \phi_{k}
$$

is valid in $B(H)$. The difference to the present paper is that, in [3], the expansion is not in eigenvalues and eigenvectors/principal vectors of the compact operator $T$ itself. 


\section{Conclusions}

In this paper it is shown that expansions in series of eigenvectors valid for symmetric linear compact operators and symmetric densely defined linear operators with compact inverse can be carried over to corresponding nonsymmetric operators where, in the case of general eigenvalues, the expansions are in series of principal vectors. These results are all new and mean a considerable progress in the Spectral Analysis of Nonsymmetric Linear Compact Operators in a Hilbert Space. The expansions discussed in Section 7 are not in series of eigenvectors resp. principal vectors and thus are different from ours. Further, in Natural Sciences and Engineering, expansions in series of eigenvectors and principal vectors are of particular importance. Our results are applicable to general non-selfadjoint BEVPs pertinent to an ordinary differential operator of nth order and deliver even there new results when the eigenvalues are general, that is, not necessarily simple. In a special example of a differential operator of 2nd order with constant coefficients, the eigenvalues, eigenfunctions and the Green's functions are explicitly determined which also seems to be new, as far as the author is aware.

\section{Acknowledgements}

The author would like to express their sincere thanks to the editor and the anonymous reviewers for their helpful comments and suggestions.

\section{Funding}

There is no funding for this work.

\section{Availability of data and materials}

Not applicable.

\section{Competing interests}

The author declares that he has no competing interests

\section{References}

[1] N.I. Achieser, I.M. Glasmann, Theorie der linearen Operatoren im Hilbert-Raum (Theory of Linear Operators in Hilbert Space; German Translation of the Russian Original), Akademie-Verlag, Berlin, 1968.

[2] E.A. Coddington, N. Levinson, Theory of Ordinary Differential Equations, McGraw-Hill Book Company, New York Toronto London, 1955.

[3] D.K. Crane, M.S. Gockenbach, M.J. Roberts, Approximating the Singular Value Expansion of a Compact Operator, SIAM J. Numer. Anal. 58(2)(2020), 1295-1318.

[4] R.D. Grigorieff, Diskrete Approximation von Eigenwertproblemen. III: Asymptotische Entwicklungen (Discrete Approximation of Eigenvalue Problems. III: Asymptotic Expansions), Num. Math. 25(1975), 79-97.

[5] H. Heuser, Funktionalanalysis (Functional Analysis), B.G. Teubner, Stuttgart, 1975.

[6] T. Kato, Perturbation Theory for Linear Operators, Springer-Verlag, New York, 1966.

[7] K. Knopp, Theory of Functions, Parts I and II (English Translation of the German Original), Dover Publications, 1975.

[8] L. Kohaupt, Construction of a Biorthogonal System of Principal Vectors for Matrices A and $A^{*}$ with Applications to $\dot{x}=A x, x\left(t_{0}\right)=x_{0}$, J. Comput. Math. Optim., 3(3)(2007), 163-192.

[9] L. Kohaupt, Biorthogonalization of the Principal Vectors for the Matrices A and $A^{*}$ with Applications to the Computation of the Explicit Representation of the Solution $x(t)$ of $\dot{x}=A x, x\left(t_{0}\right)=x_{0}$, Appl. Math. Sci., 2(20)(2008), 961-974.

[10] W. Luther, K. Niederdrenk, F. Reutter, H. Yserentant, Gewöhnliche Differentialgleichungen, Analytische und numerische Behandlung (Ordinary Differential Equations, Analytic and Numerical Treatment), Vieweg, Braunschweig Wiesbaden, 1987.

[11] S.G. Michlin, Variationsmethoden der Mathematischen Physik (Variational Methods of Mathematical Physics; German Translation of the Russian Original), Akademie-Verlag, Berlin, 1962.

[12] E.C. Pestel, F.A. Leckie, Matrix Methods in Elastomechanics, McGraw-Hill Book Company, Inc., New York San Francisco Toronto London, 1963. 
[13] F. Stummel, Diskrete Konvergenz linearer Operatoren II (Discrete Convergence of Linear Operators, Part II), Math. Z., 120(1971), 231-264.

[14] A.E. Taylor, Introduction to Functional Analysis, John Wiley \& Sons, New York London, 1958. 